\title{
The expression of inhibitor of nuclear factor kappa-B kinase epsilon (IKK $\varepsilon$ ) in human aortic aneurysm
}

\author{
L. Zhang\#, L. Wang\#, W. Chen, Y. Xu, L. Wang, R. Iskandar, Y. Wang, X. Chen \\ Department of Thoracic and Cardiovascular Surgery, Nanjing First Hospital, Nanjing Medical University, \\ Jiangsu, People's Republic of China
}

[Received: 5 September 2016; Accepted: 17 November 2016]

Background: Aortic aneurysm (AA) is one of the most common causes of sudden death among elderly people. Although AA can be detected by non-invasive imaging techniques, there are no pharmacological treatments currently available to prevent progression at any stage of the disease. In this study we will explore the expression of inhibitor of nuclear factor kappa-B kinase epsilon (IKKE) in $A A$ and its potential underlying molecular mechanism in AA.

Materials and methods: Human aortic tissue was taken from 14 patients who underwent surgical repair of $A A$ for the AA group and another 11 patients with normal aorta who underwent aortic valve replacement surgery for the control group. After excision, we used haematoxylin-eosin staining, Masson staining, immunohistochemistry analysis and Western blot analysis to observe the expression, location and morphological changes of the IKKE, P50 and the extracellular matrix within the $A A$.

Results: In the AA group, haematoxylin-eosin staining revealed a loss of medial integrity and inflammatory cell infiltration. Masson staining confirmed the degradation of the extracellular matrix in the AA group. Immunohistochemistry analysis showed increased infiltration of inflammatory cells and up-regulation of proinflammatory cytokines in the AA group when compared to the control group. Based on immunohistochemistry and Western blot analysis, there was clearly over-expression of $I K K \varepsilon, P 50$ and MMP2 in AA group, mainly in the intrinsic aortic cells of the media.

Conclusions: The over-expression of IKKE may play an important role in the origination and progression of $A A$ and might be a vital target for their treatment. (Folia Morphol 2017; 76, 3: 372-378)

Key words: IKKE, P50, inflammation, aortic aneurysm, metalloproteinase

\section{INTRODUCTION}

Aortic aneurysm (AA) is a degenerative disease of the aorta with a natural history of progressive dilation and rupture. In the past decade, AA have been detected using colour Doppler, multislice spiral computed tomography angiography (MCSTA) and digital subtraction angiography, but no pharmacological therapy can limit their progression or prevent their rupture [16]. AA are char-

Address for correspondence: X. Chen, PhD, MD, Professor and Director, Department of Thoracic and Cardiovascular Surgery, Nanjing First Hospital, Nanjing Medical University, Changle Road 68, Nanjing 210006, Jiangsu, P.R. of China, tel/fax: 86-25-52247821, e-mail: stevecx@njmu.edu.cn

\# Both authors contributed equally to the work. 
acterised by the medial degeneration, and investigations of the pathogenesis of AA focus mainly on the effects of hypertension, age, and apoptotic pathway activation, which finally results in the apoptosis of smooth muscle cells and degradation of the extracellular matrix $[11,15]$. Hinterseher et al. [4] showed that gene such as GATM, FOSB, ADCY7 and proteins such as CXCR4, PLEK, and GATM participated in the development of AA using a custom-designed "AA-chip", Western blot and immunohistochemical staining. However, the precise underlying aetiology of AA remains unclear.

$A$ recent study showed that inhibitor of nuclear factor kappa-B kinase epsilon (IKKE) is related to vascular inflammation, which accounts for its important role in the atherosclerosis, and it also has a potential relationship with the hypertension [13]. The role of IKK $\varepsilon$ in the development of atherosclerosis was further verified in our previous investigation, and recent studies have confirmed that atherosclerosis is a risk factor for AA $[1,6,13]$. Matrix metalloproteinase-2 (MMP-2), a downstream of $I K K \varepsilon$, is widespread and responsible for extracellular collagen degradation and remodelling [8]. Therefore, we assumed that IKK $\varepsilon$ might play a role in the development and progression of $A A$.

In this study, we examined the activation of IKK $\varepsilon$ and its related downstream factors in aortic tissues from the patients in the aortic aneurysm group and the control group, to observe whether IKK $\varepsilon$ has an underlying role in $\mathrm{AA}$.

\section{MATERIALS AND METHODS}

Patient enrolment and aortic specimen collection

The study conforms to the principles outlined in the Declaration of Helsinki. The trial was approved by the ethics committee of Nanjing Medical University affiliated Nanjing First Hospital and each patient provided written informed consent. Between 2011 and 2013, 102 patients were admitted to in Nanjing First Hospital for the treatment of the AA. Patients meeting the criteria for the study were then divided into one of the groups (AA group and control group). We randomly selected 14 patients who underwent open surgical repair of $A A$ to avoid bias for the $A A$ group and another 11 patients who underwent aortic valve replacement surgery with normal aortas for the control group. Patients were excluded if they had a history of heritable connective tissue disease, infected pseudoaneurysms, post-traumatic AA and peripheral artery diseases. The patient age, gender and characteristics were also obtained. The study
Table 1. Clinical characteristics of the aortic aneurysms patients undergoing emergent surgical repair and the control group of patients

\begin{tabular}{lcc}
\hline Item & $\begin{array}{c}\text { Control group } \\
\text { (n= 11) }\end{array}$ & $\begin{array}{c}\text { Patients } \\
(\mathbf{n}=14)\end{array}$ \\
\hline Age [years] & $70.0 \pm 8.8$ & $69.7 \pm 7.7$ \\
Sex (male/female) & $9 / 2$ & $11 / 3$ \\
Hypertension & $2(18.2 \%)$ & $2(14.2 \%)$ \\
Hyperlipidaemia & $5(45.4 \%)$ & $4(28.6 \%)$ \\
Diabetes & $2(18.2 \%)$ & $3(21.4 \%)$ \\
Coronary artery disease & $1(9.09 \%)$ & $0(0.00 \%)$ \\
Chronic kidney disease & $0(0.00 \%)$ & $0(0.00 \%)$ \\
\hline
\end{tabular}

population characteristics are summarised in additional information Table 1. Samples of human aortic tissue were excised from the area of the aneurysm during surgery. After excision, we used cold saline to store the samples and removed thrombi. The aortic specimens were either snap-frozen in liquid nitrogen or fixed in $4 \%$ formalin until analysis.

\section{Haematoxylin and eosin staining}

After $48 \mathrm{~h}$ in $4 \%$ formalin, the tissue samples were embedded in paraffin and then sliced into serial aortic sections ( $3 \mu \mathrm{m}$ thickness) with a slicing machine before being stained with haematoxylin and eosin (H\&E) and Masson trichrome.

\section{Masson trichrome stain}

The fixed aorta samples were dehydrated, and paraffin-embedded, and then sectioned at a thickness of $3 \mu \mathrm{m}$. The sections were used for Masson trichrome staining and were observed with an Olympus BX-URA2 camera.

\section{Western blotting analysis}

The frozen aortic tissue samples were homogenised, and the proteins were collected and then separated by SDS-PAGE. Then, the proteins were transferred to polyvinylidene fluoride (PVDF) membranes (Millipore, Billerica, MA, USA), which were washed twice for $10 \mathrm{~min}$ each in Tris-buffered saline (TBS) with Tween ${ }^{\circledR}$ diluted 1:1000 (TBST; Promega, Madison, WI, USA) and then blocked with TBS containing $5 \%$ non-fat milk powder for $1 \mathrm{~h}$. Next, the following primary antibodies were incubated with the membranes in TBS with Tween plus 5\% milk overnight at $4^{\circ} \mathrm{C}$ : anti-IKKE (1:500; Novus Biologi- 
Table 2. The details of antibodies

\begin{tabular}{|c|c|c|c|c|c|c|}
\hline Antibody name & $\begin{array}{l}\text { Catalogue } \\
\text { code }\end{array}$ & Lot No. & $\begin{array}{c}\text { Primary/secondary } \\
\text { antibody }\end{array}$ & Company & $\begin{array}{l}\text { The experimental } \\
\text { subjects }\end{array}$ & Use for \\
\hline Anti- IKKE & AF3199 & & Primary antibody & Novus Biological & & Western blot \\
\hline Anti-P50 & sc-114 & \#H2178 & Primary antibody & Santa Cruz & Human breast tumour & $\begin{array}{l}\text { Western blot, immunohisto- } \\
\text { chemical staining }\end{array}$ \\
\hline Anti-GAPDH & sc-25778 & & Primary antibody & Santa Cruz & $\begin{array}{l}\text { HeLa cells, human } \\
\text { lung tissue }\end{array}$ & Western blot \\
\hline Anti-MMP2 & ab110186 & GR52940-7 & Primary antibody & Abcam & $\begin{array}{l}\text { Human mammary } \\
\text { cancer tissue }\end{array}$ & $\begin{array}{l}\text { Western blot, immunohisto- } \\
\text { chemical staining }\end{array}$ \\
\hline Goat-anti-rabbit lgG & ZB-2301 & 127760 & Secondary antibody & ZSGB-BIO & & $\begin{array}{l}\text { Western blot, immunohisto- } \\
\text { chemical staining }\end{array}$ \\
\hline Anit-CD68 & ab125212 & GR77386-17 & Primary antibody & Abcam & Murine spleen tissue & $\begin{array}{l}\text { Immunohistochemical } \\
\text { staining }\end{array}$ \\
\hline Anti-IL-6 & ab6672 & GR106735-3 & Primary antibody & Abcam & $\begin{array}{l}\text { THP1 cell, human lung, } \\
\text { mouse mesenchymal } \\
\text { SC cells }\end{array}$ & $\begin{array}{l}\text { Immunohistochemical } \\
\text { staining }\end{array}$ \\
\hline
\end{tabular}

cals, Littleton, USA), anti-p50 (1:500; Santa Cruz Biotechnology, Santa Cruz, CA), anti-GAPDH (1:5000; Santa Cruz Biotechnology) and anti-MMP-2 (1:500; Abcam). The next day, after washing with TBST four times for $10 \mathrm{~min}$ each time at room temperature, the PVDF membranes were incubated with proportionally diluted peroxidase-conjugated goat anti-rabbit secondary antibodies (1:1000; Beijing ZhongShan Biotechnology Co.) for $1 \mathrm{~h}$. Furthermore, for the specific proteins, we used an ECL reagent (GE Healthcare, Piscataway, NJ, USA) to detect and capture images on Hyperfilm (Amersham, GE Healthcare). Ultimately, we evaluated the results using Image J software [1] for semi-quantitation of the mean grey value of each blot. All the details of antibodies are in additional information Table 2.

\section{Immunohistochemical staining}

The perfusion-fixed aortas were embedded, cut into cross sections $(3 \mu \mathrm{m})$ and rehydrated in graded alcohol for morphological analysis. To prevent the non-specific binding of antibodies and to eliminate endogenous peroxidase activity, the sections were incubated in buffered normal horse serum and treated with 3\% hydrogen peroxide for $15 \mathrm{~min}$. Then, we incubated the sections with anti-CD68 (1:200; Abcam), anti-P50 (1:100; Santa Cruz), anti-MMP (1:500; Abcam), and anti-IL-6 (1:500; Abcam) primary antibodies for $14 \mathrm{~h}$. The sections were incubated with horseradish peroxidase (HRP)-conjugated goat anti-rabbit lgG (Beijing Zhongshan Biotechnology Co., Beijing, China) for $1 \mathrm{~h}$ at $37^{\circ} \mathrm{C}$ in a humidified box. Subsequently, upon application of the substrate diaminobenzidine (DAB, Beijing Zhongshan Biotechnology Co.), the signal for each antibody appeared. The sections were then counterstained, and the differences in these signals were determined. As a negative control, the primary antibodies were omitted from the reaction sequence. The data were recorded using an Olympus BX-URA2 camera via photomicrographs. All the details of antibodies are in additional information Table 2.

\section{Statistical analysis}

All continuous variables are presented as the means \pm standard errors. Statistical significance was defined as a $p$-value of 0.05 . The analyses were performed using SPSS17.0 (SPSS Inc, Chicago, IL, USA) software. Categorical variables were compared using an independent-sample $t$ test.

\section{RESULTS}

\section{Morphological differences of} the aortic tissue samples

The results of the H\&E staining showed that the patients in the AA group had a damaged and thinner medial layer with disordered smooth muscle cells and a fibrin clot in the edge between the inner media and intima. Furthermore, the entire aortic vessel wall showed neutrophil infiltration, particularly in the medial layer. By contrast, there was no neutrophil infiltration in the control group, and the aortic morphology was normal (Fig. 1A). 
A

Control

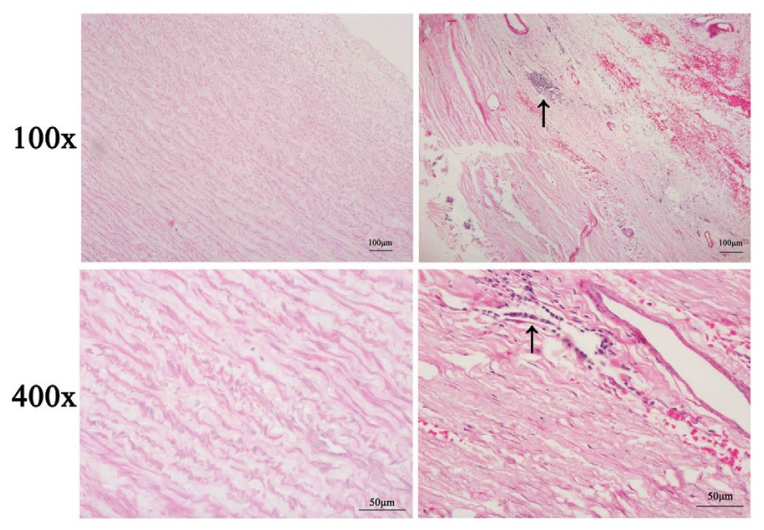

B

Control

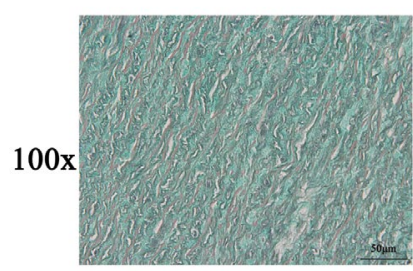

Aneurysm

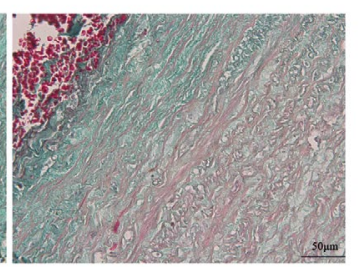

Figure 1. Morphological changes in the aortic aneurysm (AA) group and control group; A. Haematoxylin-eosin staining of the aortic vessel wall of AA and the normal aortic media of the control group. The integrity of the media was maintained and smooth muscle cells were arranged in an orderly fashion in the control group. In the aortic vessel wall of the aneurysm group, there were disarranged smooth muscle cells in the media, representing the infiltration of neutrophils throughout the entire aortic vessel wall, particularly in the media (the above are magnification $\times 100$, the down are magnification $\times 400$, arrow to indicate the inflammatory infiltrations); B. Changes in the extracellular proteins between the AA and control groups. Green represents collagen fibres, whereas red represents muscle fibres. The collagen of the aorta was significantly degraded in the AA group compared to the control group (magnification $\times 400$ ).

The Masson trichrome stain is mainly used for the identification of collagen fibres (green) and the muscle fibres (red). As it is shown, the collagen of the aorta was degraded in the AA group compared to the control group (Fig. 1B).

\section{Inflammatory infiltration in AA}

To assess inflammatory infiltration, we used immunostaining for macrophages (CD68) and the proinflammatory cytokine interleukin 6 (IL-6). Our results showed that CD68 and IL- 6 were observed throughout the tunica media at a uniformly distributed density in the AA group while the control group showed no of such expression (Fig. 2).

\section{Expression of IKK $\varepsilon$ in AA}

Western blot analysis showed a significant difference in the expression of IKK $\varepsilon$ (independent $t$ test, $t=5.878, d f=18, p<0.01$ ) between two groups (Fig. 3A, B). Similar results were also obtained using immunohistochemical staining for IKK $\varepsilon$. In addition, the area of increased expression of IKK $\varepsilon$ was primarily in the medial layer of the aortic vessel wall in AA patients (Fig. 3C).

\section{Expression of P50 in AA}

Western blot analysis showed the expression of P-P50 (independent $t$ test, $t=10.561, d f=18$, $p<0.01$ ) was significantly increased in the AA group compared to the control group (Fig. 4A). Immunohistochemical staining analysis was used to assess the activity and location of P-P50, and the results showed that P-P50 was located in the nuclei of stromal cells.

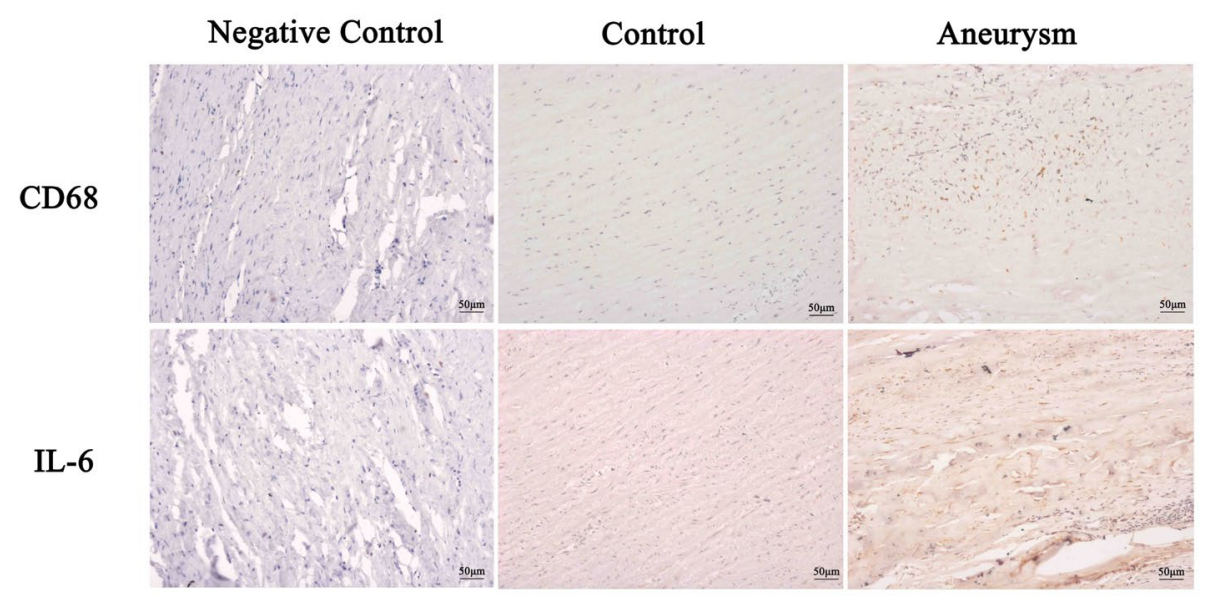

Figure 2. Immunohistochemistry staining for interleukin 6 (IL-6) and CD68 showed their increased expression in the aortic aneurysm group compared to the control group (magnification $\times 400$ ). 
A

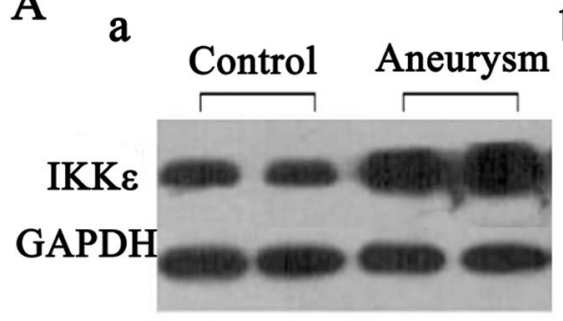

b

B
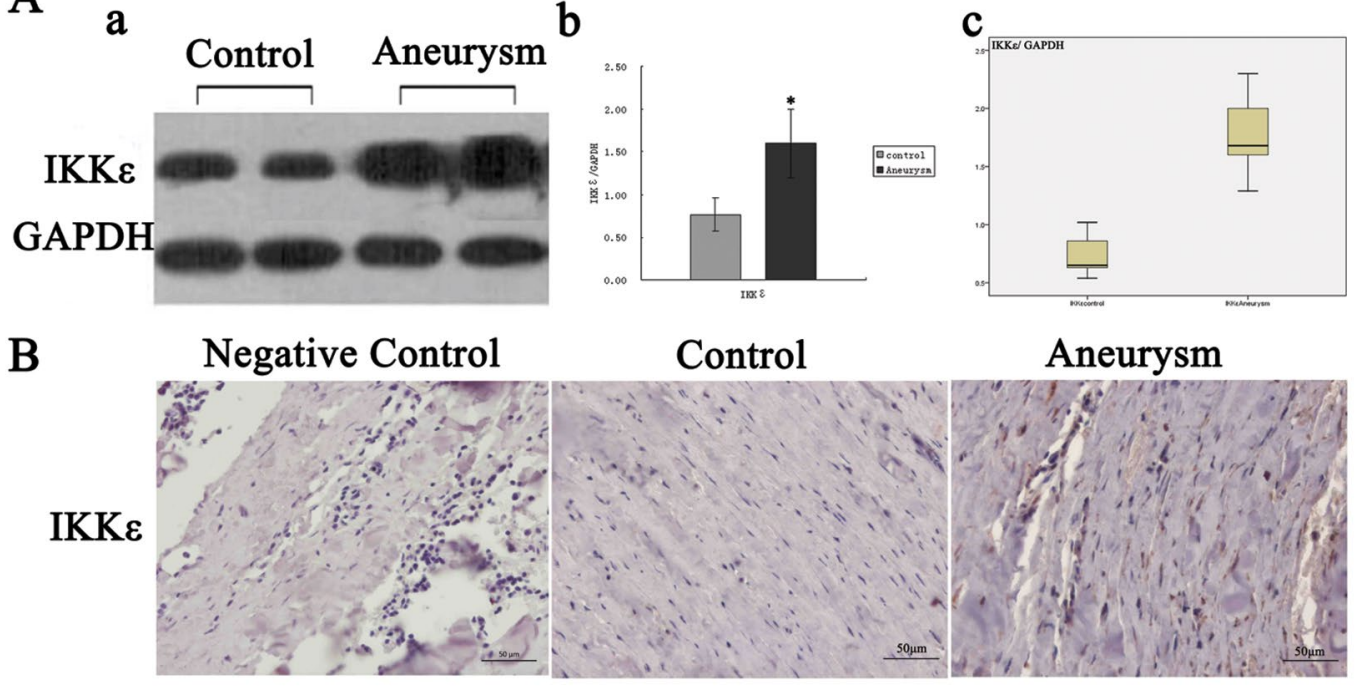

Figure 3: Protein expression and localisation of IKK $\varepsilon$ in the aortic aneurysm (AA) group and control group; A. The protein level of IKKE was measured by Western blotting; a. Western blotting of IKK $\varepsilon$ and GAPDH; $\mathbf{b}$. The expression of IKKE in the aortic vessel wall after normalisation to GAPDH was significantly increased in the AA group compared to the control group. The values are the means \pm standard error; ${ }^{*} p<0.01$; c. Box-and-whisker plots of IKK $\varepsilon ; \mathbf{B}$. Immunohistochemistry staining also showed that IKK $\varepsilon$ were up-regulated in the AA group when compared with the control group, and the expression of IKKE was mainly distributed in the medial area of the aortic vessel wall (magnification $\times 400$ ).

A
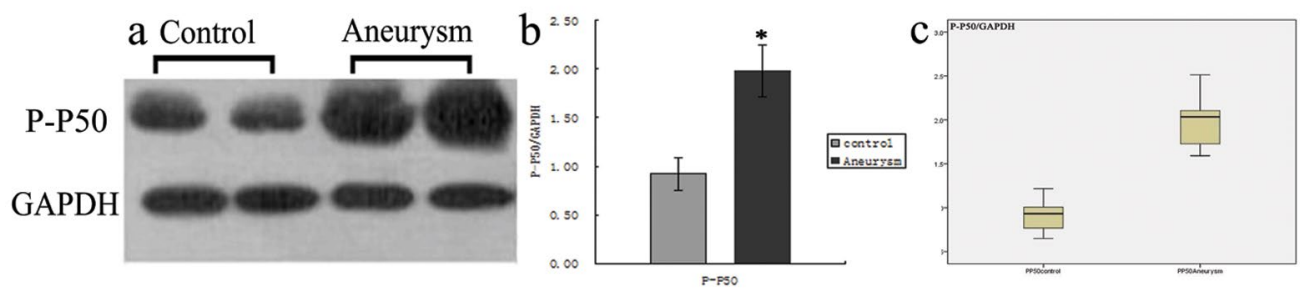

B

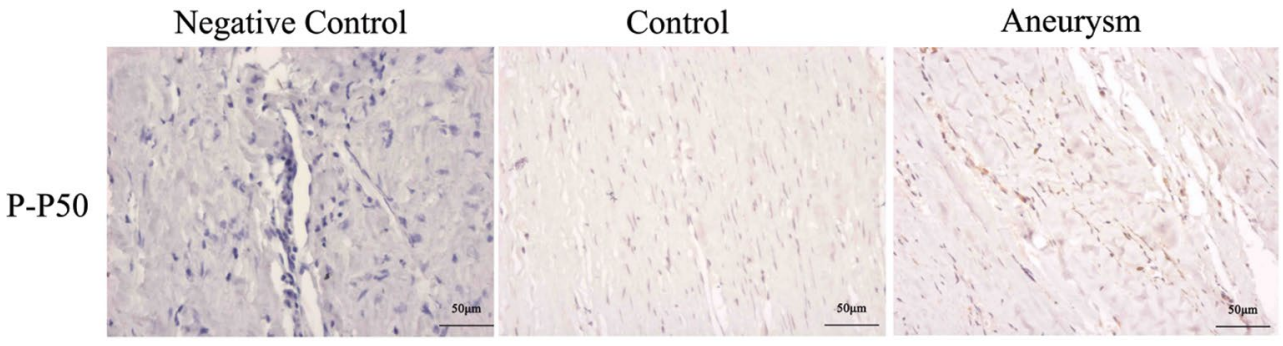

Figure 4. Protein expression and localization of P50 determined in the aortic aneurysm (AA) group and control group; $\mathbf{A}$. The protein level of P50 was measured by Western blotting; $\mathbf{a}$. Western blotting of P50 and GAPDH; $\boldsymbol{b}$. The expression of P50 in the aortic vessel wall after normalisation to GAPDH was significantly increased in the AA group compared to the control group. The values are the means \pm standard error; * $p<0.01$; c. Box-and-whisker plots of P50; B. Representative images showed the up-regulated expression of P50 in the aorta of the AA group compared to the control group, which was spread throughout the entire media and adventitia, particularly in the inner media at the border between the intima and media (magnification $\times 400$ ).

These results indicate that the protein is biologically active in the AA group (Fig. 4B).

\section{Expression of MMP-2 in AA}

To verify the expression and location of MMP-2, Western blot analysis and immunostaining were used to detect MMP-2. Western blot analysis showed that the expression of MMP-2 was significantly increased in the AA group compared to the control group (independent $t$ test, $t=5.221$, $\mathrm{df}=18, \mathrm{p}<0.01$ ) (Fig. 5A). Immunostaining confirmed that MMP-2 was primarily distributed in the media in the AA group but not in the control group (Fig. 5B). 


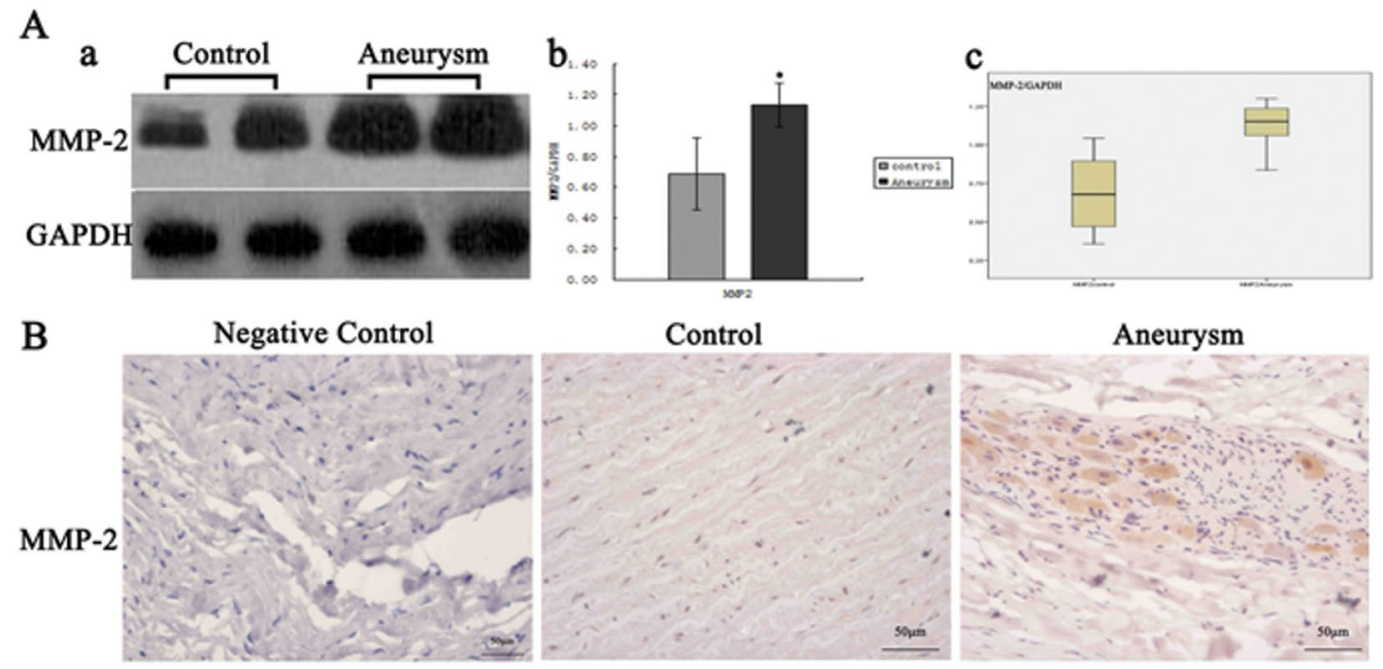

Figure 5. Protein expression, localisation and activity of MMP-2 in the aortic aneurysm (AA) and control groups; $\mathbf{A}$. The protein level of MMP-2 was measured by Western blotting; a. Western blotting of MMP-2; $\mathbf{b}$. The expression level of MMP-2 in the aortic vessel wall after normalisation to GAPDH was significantly increased in the AA group compared to the control group. The values are the means \pm standard error; ${ }^{*} p<0.01$; c. Box-and-whisker plots of MMP-2; B. The representative images show the upregulated expression of MMP-2 in the aorta of the AA group compared to the control group, which was spread throughout the entire media and adventitia, particularly in the inner media at the border between the intima and media (magnification $\times 400$ ).

\section{DISSCUSION}

Aortic aneurysm is a progressive disease of the aorta and is mainly associated with smoking, advanced age, male sex and atherosclerosis [3]. Existing evidence shows that the occurrence of $A A$ is associated with the degradation of the tunica medial and an inflammatory reaction $[7,12]$. Histological analysis has shown that the extracellular matrix degeneration is characterised by disruption and degradation of medial elastin and gross medial collagen deposition, whereas inflammation is exemplified by inflammatory cell infiltration of the aortic wall. We observed the same results in our experiment. The integrity of the tunica media was disrupted and there was a thinner medial thickness in the AA group, which accompanied with main pathological findings of this disease.

The main pathological characteristics of the experimental group included neutrophil infiltration and disordered of the smooth muscle cells which could be observed. In addition, we also detected increased infiltration of macrophages by immunohistochemical staining for CD68. IL-6 is a downstream factor of the nuclear factor $-\kappa B$ cascade, which is secreted at high levels in human AA disease [5, 9]. It has also been identified as an independent biomarker for severe coronary artery disease [2], and is associated with the risk of aneurysm rupture [10]. There was a significance difference between the AA group and the control group in the expression of IL-6, which was mainly located in the medial area in the AA group.
$\mathrm{IKK} \varepsilon$ influences nuclear factor- $\kappa \mathrm{B}$ signalling and induces inflammation, cell proliferation, tumour cell survival and cell transformation [14]. A significant overexpression of IKK $\varepsilon$ was observed in the AA group compared to the control group. Together with the Western blotting results, immunohistochemical staining showed upregulation of IKK $\varepsilon$ in the AA group, which was distributed particularly in the medial layer. These results indicate that the pathological and morphological changes of the AA might be due to the upregulation of IKK $\varepsilon$.

We assessed changes in P50 in the AA group to determine whether the activation of IKKE promotes $A A$ through the activation of $\mathrm{P50}$. Our study demonstrated that the expression of total and phosphorylated P50 in the aortic vessel wall was increased in the AA group compared to the control group, suggesting a role of P50 in the progression and formation of AA. MMP-2 plays an important role in the degradation of extracellular proteins and the remodelling of the extracellular matrix. The expression of MMP-2 was significantly increased in the AA group compared to the control group.

In this study, immunohistochemistry was used to visualise the localisation of $I K K \varepsilon, \mathrm{P} 50$ and MMP-2. Our results revealed that IKK $\varepsilon$, P50 and MMP-2 were upregulated in the aortic tissue of the AA group compared to the control group.

Based on these results, our study suggests that the expression of IKK $\varepsilon$ and P50 signalling may regu- 
late the transcription of diverse genes encoding cytokines (IL-6), which could lead to the infiltration of macrophages and the secretion of MMPs. Moreover, the extracellular matrix of the medial layer was degenerated, which resulted in the formation of the AA. This study also provides us a deeper insight of the AAs at the molecular level, which may lead to a better understanding and future treatment and also prevention of patients with AA through the inhibition of IKKE.

\section{CONCLUSIONS}

The over-expression of IKK $\varepsilon$ may play an important role in the origination and progression of $A A$ and might be a vital target for their treatment.

\section{Sources of funding}

This study was supported by grants from the National Natural Science Foundation of China (No. 81370259).

\section{Acknowledgements}

Dr. Xin Chen is a fellow at the Collaborative Innovation Centre for Cardiovascular Disease Translational Medicine.

\section{REFERENCES}

1. Cao C, Zhu Y, Chen W, et al. IKKepsilon knockout prevents high fat diet induced arterial atherosclerosis and NF- $\kappa$ B signaling in mice. PLoS One. 2013; 8(5): e64930, doi: 10.1371/journal. pone.0064930, indexed in Pubmed: 23741427.

2. Cheuk BLY, Cheng SWK. Can local secretion of prostaglandin E2, thromboxane B2, and interleukin- 6 play a role in ruptured abdominal aortic aneurysm? World J Surg. 2008; 32(1): 55-61, doi: 10.1007/s00268-007-9279-9, indexed in Pubmed: 17992560.

3. Golledge J, Muller J, Daugherty A, et al. Abdominal aortic aneurysm: pathogenesis and implications for management. Arterioscler Thromb Vasc Biol. 2006; 26(12): 2605-2613, doi: 10.1161/01.ATV.0000245819.32762.cb, indexed in Pubmed: 16973970.

4. Hinterseher I, Erdman R, Elmore JR, et al. Novel pathways in the pathobiology of human abdominal aortic aneurysms. Pathobiology. 2013; 80(1): 1-10, doi: 10.1159/000339303, indexed in Pubmed: 22797469.

5. Jones KG, Brull DJ, Brown LC, et al. Interleukin-6 (IL-6) and the prognosis of abdominal aortic aneurysms. Cir- culation. 2001; 103(18): 2260-2265, indexed in Pubmed: 11342474.

6. Kim YW, West XZ, Byzova TV. Inflammation and oxidative stress in angiogenesis and vascular disease. J Mol Med (Berl). 2013; 91(3): 323-328, doi:10.1007/s00109-0131007-3, indexed in Pubmed: 23430240.

7. Lenk GM, Tromp G, Weinsheimer S, et al. Whole genome expression profiling reveals a significant role for immune function in human abdominal aortic aneurysms. BMC Genomics. 2007; 8: 237, doi: 10.1186/1471-2164-8-237, indexed in Pubmed: 17634102.

8. Löffek S, Schilling O, Franzke CW. Series "matrix metalloproteinases in lung health and disease": Biological role of matrix metalloproteinases: a critical balance. Eur Respir J. 2011; 38(1): 191-208, doi: 10.1183/09031936.00146510, indexed in Pubmed: 21177845.

9. Nagase H, Visse R, Murphy G. Structure and function of matrix metalloproteinases and TIMPs. Cardiovasc Res. 2006; 69(3): 562-573, doi:10.1016/j.cardiores.2005.12.002, indexed in Pubmed: 16405877.

10. Rohde LE, Arroyo LH, Rifai N, et al. Plasma concentrations of interleukin- 6 and abdominal aortic diameter among subjects without aortic dilatation. Arterioscler Thromb Vasc Biol. 1999; 19(7): 1695-1699, indexed in Pubmed: 10397687.

11. Svensjö S, Björck $M$, Gürtelschmid $M$, et al. Low prevalence of abdominal aortic aneurysm among 65-year-old Swedish men indicates a change in the epidemiology of the disease. Circulation. 2011; 124(10): 1118-1123, doi: 10.1161/CIRCULATIONAHA.111.030379, indexed in Pubmed: 21844079.

12. Thompson RW. Reflections on the pathogenesis of abdominal aortic aneurysms. Cardiovasc Surg. 2002; 10(4): 389-394, indexed in Pubmed:12359414.

13. Tsai SH, Huang PH, Peng YJ, et al. Zoledronate attenuates angiotensin II-induced abdominal aortic aneurysm through inactivation of Rho/ROCK-dependent JNK and NF-kappaB pathway. Cardiovasc Res. 2013; 100(3): 501-510, doi: 10.1093/cvr/cvt230, indexed in Pubmed: 24225494.

14. Verhelst K, Verstrepen L, Carpentier I, et al. IkappaB kinase epsilon (IKKepsilon): a therapeutic target in inflammation and cancer. Biochem Pharmacol. 2013; 85(7): 873-880, doi: 10.1016/j.bcp.2013.01.007, indexed in Pubmed: 23333767.

15. Yamashita O, Yoshimura K, Nagasawa A, et al. Periostin links mechanical strain to inflammation in abdominal aortic aneurysm. PLoS One. 2013; 8(11): e79753, doi: 10.1371/journal.pone.0079753, indexed in Pubmed: 24260297.

16. Yoshimura K, Aoki H. Recent advances in pharmacotherapy development for abdominal aortic aneurysm. Int J Vasc Med. 2012; 2012: 648167, doi:10.1155/2012/648167, indexed in Pubmed: 22957259. 